\title{
Celiac artery coverage after occlusion test during endovascular stent grafting for thoracoabdominal aortic aneurysm
}

\author{
Taro Shimazaki, MD, Satoshi Kawaguchi, MD, Yoshihiko Yokoi, MD, Kenji Koide, MD, \\ Masataka Matsumoto, MD, and Hiroshi Shigematsu, MD, Tokyo, Japan
}

Previous investigators have reported favorable results with endovascular repair of thoracoabdominal aortic aneurysms by occlusion of the celiac artery (CA). ${ }^{1,2}$ Verification of collateral blood flow to the CA, however, is important to prevent postprocedural ischemic changes in the liver, stomach, and intestines. We describe the use of a CA occlusion test to investigate the collateral blood flow from the superior mesenteric artery (SMA) to the CA in a patient undergoing endovascular stent grafting for a thoracic and thoracoabdominal aortic aneurysm.

\section{CLINICAL SUMMARY}

A 66-year-old woman with high-risk saccular aneurysms at the distal aortic arch and thoracoabdominal aorta elected to undergo stent grafting. This relatively less invasive approach was recommended because of comorbid severe

\footnotetext{
From the Department of Vascular Surgery, Tokyo Medical University, Tokyo, Japan. Disclosures: None.

Received for publication Sept 2, 2008; accepted for publication Nov 19, 2008; available ahead of print March 9, 2009.

Address for reprints: Taro Shimazaki, MD, 6-7-1 Nishishinjuku, Shinjuku-ku, Tokyo 160-0023, Japan.

J Thorac Cardiovasc Surg 2010;139:e59-62 0022-5223/\$36.00

Copyright (c) 2010 by The American Association for Thoracic Surgery doi:10.1016/j.jtcvs.2008.11.024
}

multiple myeloma and cardiac risk factors. The thoracoabdominal aortic aneurysm was located near the CA. Because there was a risk of type I endoleak as a result of the short distal neck, occlusion of the CA was deemed necessary (Figure 1). Informed consent was obtained, and the endovascular procedure was performed in the operating room with general anesthesia. The right femoral artery was exposed. Heparin sodium (3000 units) was administered intravenously, with subsequent administration to keep activated coagulation time longer than 200 seconds.

A balloon catheter (diameter $10 \mathrm{~mm}$; Boston Scientific Corporation, Boston, Mass) was advanced through the femoral artery to the CA, and the CA was occluded by inflation of the balloon. Selective digital subtraction angiography of the SMA was performed, and collateral blood flow to the CA was confirmed (Figure 2). Stent grafting was started immediately after the occlusion test.

A 400-cm $\times 0.032$-inch guidewire was introduced through the right brachial artery and advanced into the abdominal aorta. The distal end of the guidewire was caught with a snare catheter (Amplatz gooseneck snare; Microvena Corp, White Bear Lake, Minn) and picked up at the femoral artery.

A 20F sheath (Keller Timmerman Sheath; Cook Medical Inc, Bloomington, Ind) was introduced transfemorally over the guidewire with the tug of wire technique. ${ }^{3}$ A stent graft

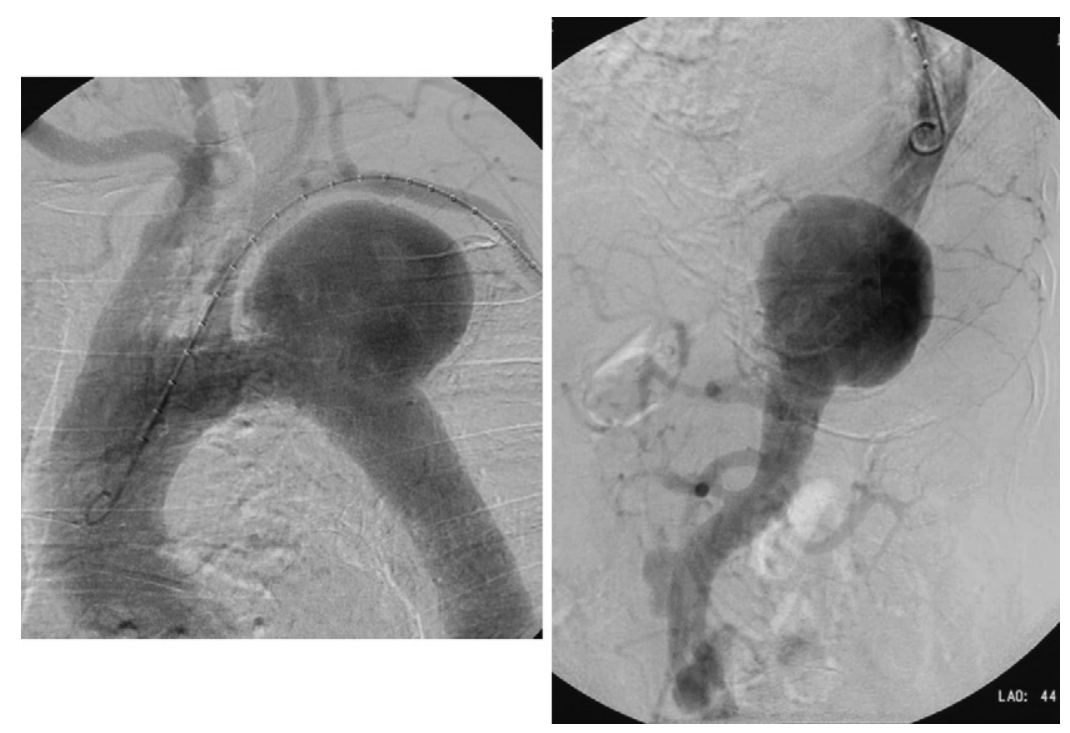

FIGURE 1. Digital subtraction angiography shows two saccular aneurysms at distal aortic arch and thoracoabdominal aorta. 


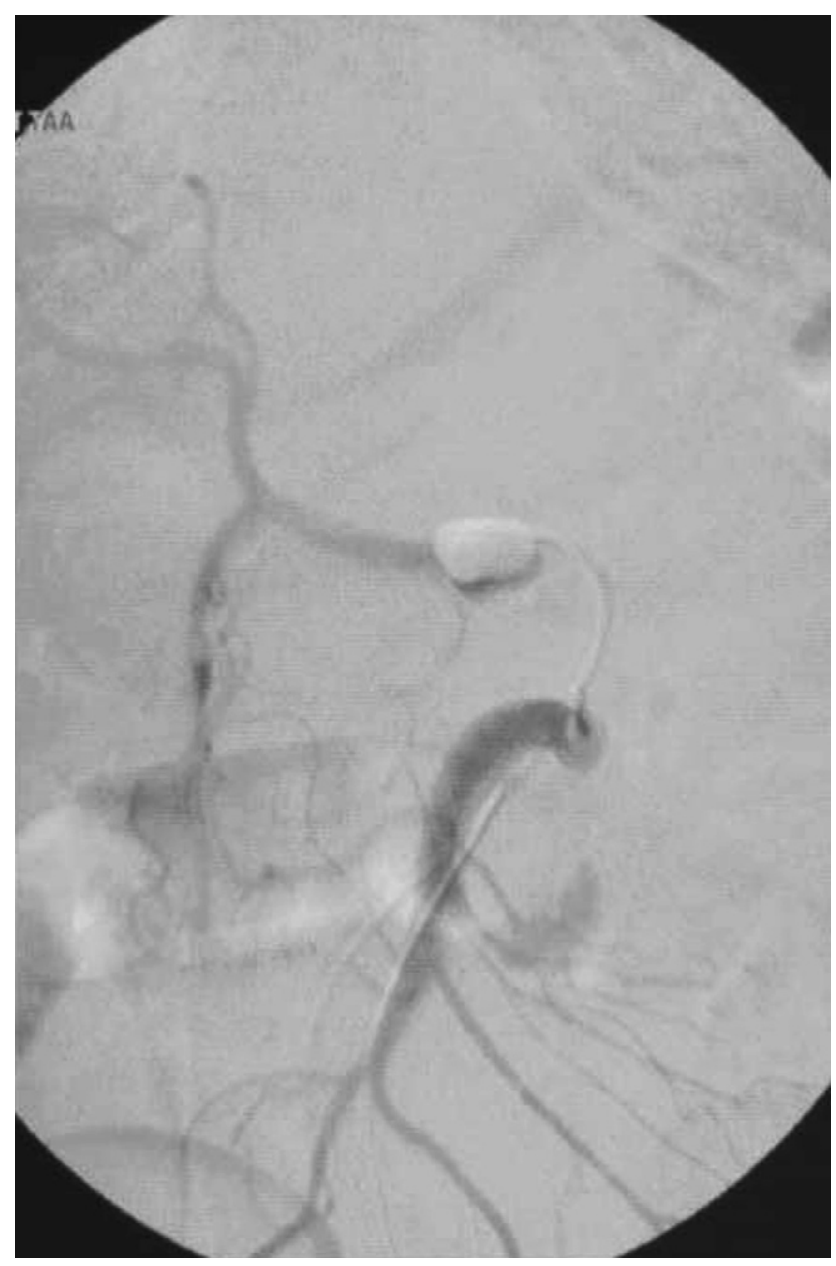

FIGURE 2. Celiac artery occlusion test verified collateral circulation between celiac and superior mesenteric arteries. was constructed from a self-expanding Gianturco $\mathrm{Z}$ stent (Cook Medical) and thin-wall woven polyester fabric (Ube woven-graft; Ube Industries, Ube, Japan). The first stent graft (diameter $34 \mathrm{~mm}$, length $170 \mathrm{~mm}$ ) was deployed in the distal arch aneurysm, and the second stent graft (diameter $30 \mathrm{~mm}$, length $195 \mathrm{~mm}$ ) was deployed in the thoracoabdominal aortic aneurysm. Postprocedural digital subtraction angiography showed collateral blood flow to CA from SMA without endoleak in either aneurysm (Figure 3).

After the operation, the patient had severe nausea and could not maintain adequate oral intake. This remitted after the third postoperative week, and the patient was discharged 37 days after stent grafting. Neither renal failure nor ischemic colitis occurred, and there were no postoperative changes in liver function. A computed tomographic scan at the third postoperative month showed exclusion of the aneurysm and confirmed spleen viability (Figure 4).

\section{DISCUSSION}

Endovascular repair of a thoracoabdominal aortic aneurysm can be complicated, and preservation of blood flow of branched vessels (eg, CA, SMA) is important. Although surgical bypass or the use of a branched stent graft may be more reliable for the management of a thoracoabdominal aortic aneurysm, such procedures are more invasive and are associated with higher rates of complications. In this case, a less invasive approach was elected because of the patient's poor underlying health (advanced multiple myeloma).

Vaddineni and colleagues ${ }^{1}$ reported outcomes after CA coverage during stent grafting and described the necessity for preprocedural angiographic verification of collateral circulation between the CA and the SMA, as we performed in this
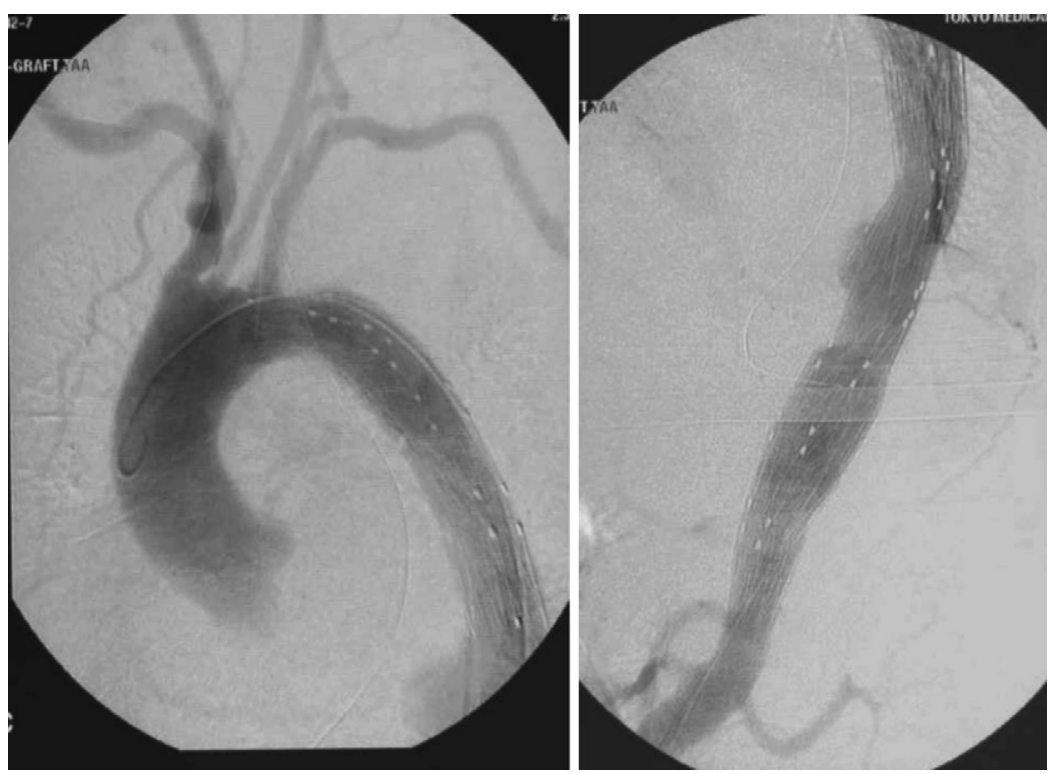

FIGURE 3. Postoperative angiography shows stent grafts excluding both aneurysms. 


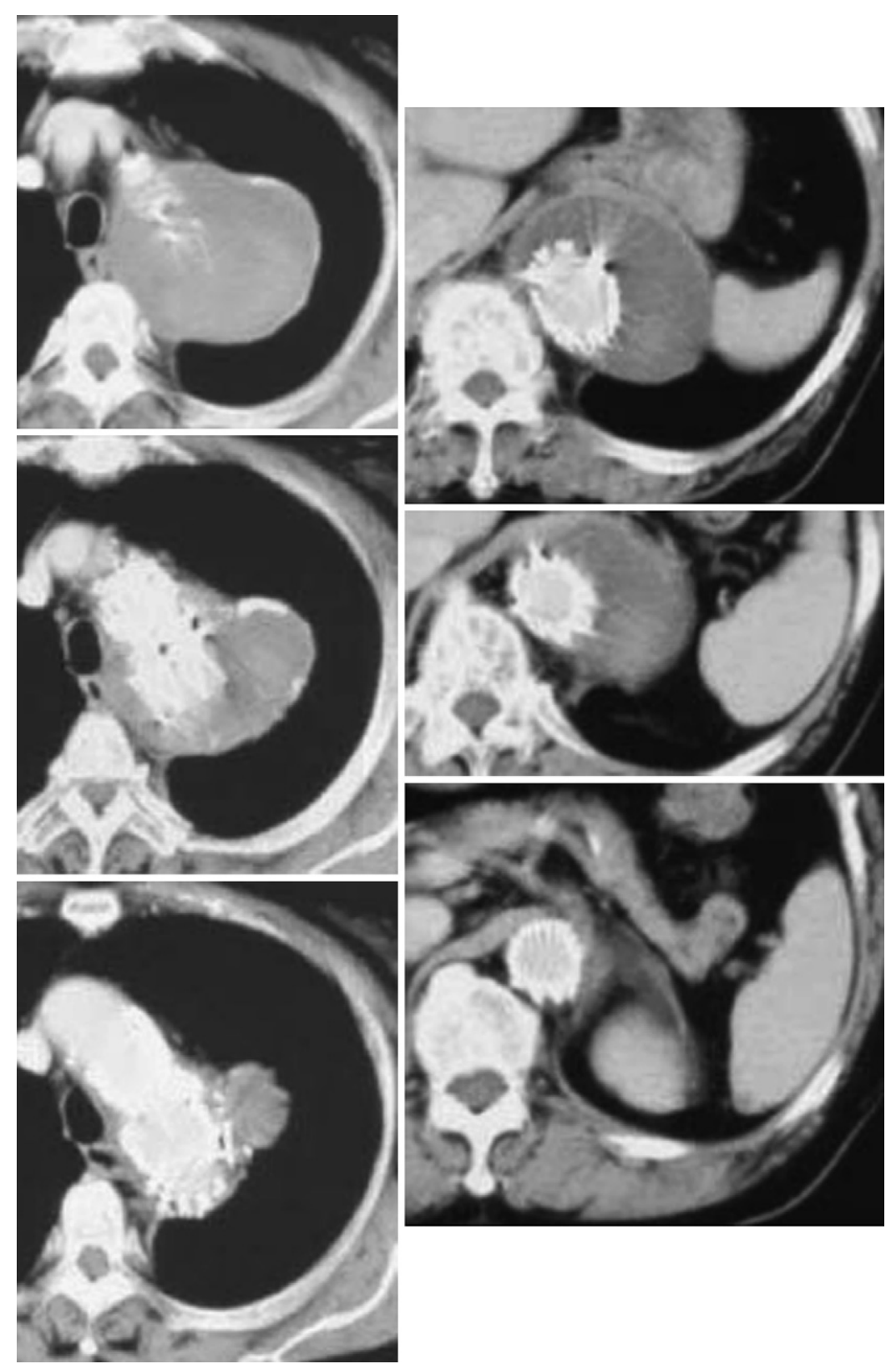

FIGURE 4. Computed tomographic scan at third postoperative month shows excluded aneurysm.

case. This was an angiographic evaluation, however, not a true functional evaluation. It is therefore unclear whether conclusion of the CA contributed to the patient's postoperative nausea.

According to our strategy, we performed the bypass only after the absence of collateral blood flow was confirmed by a CA occlusion test. To avoid severe complications as a result of the invasiveness of the bypass operation, it is important to perform a CA occlusion test in such cases.
In conclusion, we describe here the successful use of a CA occlusion test to investigate the collateral blood flow from the SMA to the CA in a patient undergoing endovascular stent grafting for a thoracic and thoracoabdominal aortic aneurysm. This testing is not a true functional evaluation of the implications of CA occlusion, however, and any patient undergoing this procedure should be observed carefully for potential ischemic complications. 


\section{References}

1. Vaddineni SK, Taylor SM, Patterson MA, Jordan WD. Outcome after celiac artery coverage during endovascular thoracic aortic aneurysm repair: preliminary results. J Vasc Surg. 2007;45:467-471.

2. Waldenberger P, Bendix N, Petersen J, Tauscher T, Glodny B. Clinical outcome of endovascular therapeutic occlusion of celiac artery. J Vasc Surg. 2007;46:655-661.
3. Ishimaru S, Kawaguchi S, Koizumi N, Obitsu Y, Ishikawa M. Preliminary report on prediction of spinal cord ischemia in endovascular stent graft repair of thoracic aortic aneurysm by retrievable stent graft. J Thorac Cardiovasc Surg. 1998;115: 811-818.

\title{
False aneurysm with aortopulmonary shunt after replacement of the ascending aorta
}

\author{
Wilko Reents, MD, ${ }^{\mathrm{a}}$ Werner Kenn, MD, ${ }^{\mathrm{b}} \mathrm{Jörg}$ Babin-Ebell, MD, ${ }^{\mathrm{c}}$ Rainer G. Leyh, MD, PhD, and \\ Armin Gorski, MD, ${ }^{\text {a }}$ Würzburg and Bad Neustadt, Germany
}

The development of a false aneurysm with an aorto-pulmonary shunt is an extraordinary, uncommon, and lifethreatening condition. Surgical repair is inevitable, yet the operative approach is hampered by several pitfalls. This report describes the tailored surgical treatment of a mediastinal oseudoaneurysm with an aorto-pulmonary shunt arising after ascending aortic replacement.

\section{CLINICAL SUMMARY}

Five years after supracoronary replacement of the ascending aorta for acute aortic dissection, a 61-year-old woman had new-onset, rapidly progressive dyspnea. At presentation, she had orthopnea and peripheral edema. Auscultation revealed a continuous diastolic-systolic murmur and diminished basilar breath sounds. Chest radiography showed bilateral pleural effusions and interstitial fluid accumulation. Echocardiography demonstrated a moderate aortic valve insufficiency with prolapse of the right coronary cusp, a periaortic fluid accumulation, and the presence of a shunt between the ascending aorta and the pulmonary artery. Magnetic resonance imaging confirmed the presence of an aortopulmonary shunt (pulmonary/systemic perfusion ratio of 1.89) and demonstrated a large false aneurysm with extrav-

From the Departments of Cardiothoracic Surgery ${ }^{\mathrm{a}}$ and Radiology, ${ }^{\mathrm{b}}$ University Hospital Würzburg, Würzburg, Germany and the Department of Cardiovascular Surgery, Cardiovascular Clinic Bad Neustadt, ${ }^{\mathrm{c}}$ Bad Neustadt, Germany.

Disclosures: None.

Received for publication Sept 22, 2008; accepted for publication Nov 19, 2008; available ahead of print Feb 9, 2009.

Address for reprints: Wilko Reents, MD, Department of Cardiothoracic Surgery, University Hospital Würzburg, Oberdürrbacher Strasse 6, 97080 Würzburg, Germany. (E-mail: wilko.reents@herzchirurgie.de).

J Thorac Cardiovasc Surg 2010;139:e62-3 0022-5223/\$36.00

Copyright (C) 2010 Published by Elsevier Inc. on behalf of The American Association for Thoracic Surgery

doi:10.1016/j.jtcvs.2008.11.022 asation of contrast media at the level of the sinotubular junction (Figure 1).

Institution of cardiopulmonary bypass with cannulation of the right carotid artery and the femoral vein was performed before sternotomy. Systemic cooling was limited to a perfusion temperature of $28^{\circ} \mathrm{C}$. Resternotomy was uneventful, and the false aneurysm was covered with pericardium. Dehiscence of the suture line between the prosthesis and the noncoronary sinus had occurred, with visible aortic tissue necrosis and remnants of previously applied BioGlue (CryoLife Inc, Kennesaw, Ga). Additionally, there was a defect at the origin of the right pulmonary artery. The necrotic tissue was excised, and the defect in the pulmonary artery and aortic sinus was closed with bovine pericardium. The aortic valve showed degenerative changes and was replaced.

Pulmonary edema developed postoperatively. Fluid management and mechanical ventilation with positive endexpiratory pressure led to rapid recovery, and the patient was extubated 12 hours after the operation. The further postoperative course was uneventful, and the patient was discharged on the 10th postoperative day. There was no growth of bacteria or fungi from samples of the necrotic aortic tissue.

\section{DISCUSSION}

False aneurysm of the thoracic aorta is a rare event. Although development after trauma or infection has been described, previous surgery is the antecedent event in most cases. ${ }^{1}$ The incidence of pseudoaneurysm formation after cardiac surgery is not known but is estimated to be less than $0.5 \%{ }^{1}$ Sites of reduced resistance include previous anastomotic sites or incisions, where transmural disruption of the aortic wall is facilitated. Possible mechanisms include infection, technical shortcomings, and intrinsic disease of the aortic wall.

The operative management in this case had to overcome four principal challenges: (1) free rupture with 